\title{
Design and Characterization of Air Coupled Ultrasonic Transducers Based on MUMPs
}

\author{
Octavio, A, Martín, C.J, Gómez- \\ Ullate, Y, Martínez, O, Gómez- \\ Ullate, L, Montero de Espinosa, F \\ IA / IAI \\ CSIC \\ Madrid, Spain
}

\author{
Gatta, P \\ Dipartimento di Ingegneria \\ Elettronica \\ Università degli Studi Roma Tre \\ Rome, Italy
}

\author{
Domínguez, M \\ Electronic Engineering Department \\ UPC \\ Barcelona, Spain
}

\begin{abstract}
This paper deals with the design and characterization of several capacitive micromachined ultrasonic transducer (cMUT) cells for the future design of an air-coupled transducer for non destructive testing (NDT). Each design was manufactured using the multi-user MEMS process (MUMPs). Special boundary conditions were used to obtain high efficiency: two opposite sides of the $150 \mu \mathrm{m}$ square shaped membranes are free while the other sides are anchored to a fixed layer of the process. Using these designs a large displacement of the membrane and a higher surface contact is obtained in spite of their mechanical losses.
\end{abstract}

Keywords-component; cMUT; MUMPs; NDT

\section{INTRODUCTION}

In ultrasonic NDT applications, there is an increasing interest on substituting conventional immersion techniques by air-coupling inspection [1]-[2]. Air-coupled piezoelectric transducers up to $2 \mathrm{MHz}$ have been designed and are commercially available. However, transducers based on microelectro-mechanical systems (MEMS) can be more efficient, besides MEMS facilitate the fabrication of complex apertures such as air coupled ultrasonic 2D arrays. In addition, due to the frequencies in air NDT are relatively low, the use of a multiuser MEMS process (MUMPs) can be justified, as it is a simple and cost effective manufacture process. Nevertheless, there is a notable lack of sensitivity which needs to be improved. The thickness of the membranes that can be fabricated following this process $(2 \mu \mathrm{m})$ produce a sensitivity lower than if thinner membranes be used $(0.5 \mu \mathrm{m}$ thick membrane of current capacitive micromachined ultrasonic transducers - cMUTs [3][4]).

This paper deals with the characterization and the finite element modelling (FEM) of several designs of cMUT cells for ultrasonic air coupled transducers to be fabricated with the MUMPS process. Five different typologies were studied to optimize the sensitivity of the transducer. The models were developed following the design rules of the MUMPs manufacture process: Polysilicon layers having $\sim 2 \mu \mathrm{m}$ thickness and $150 \mu \mathrm{m}$ cells size were the basis of the designs. As this process uses doped polysilicon as the structural material, it is not necessary the use of a metallization to make the devices work. However, one design was manufactured with a gold electrode to view the effects of the metallization of the polysilicon.

To increase the cell sensitivity, special boundary conditions were used. Two opposite sides of the membrane are free while the other edges of each cell are anchored to a fixed layer of the design (F-A-F-A). This becomes in a bigger surface contact and a large membrane displacement improving the performance of the device. A design with a square shaped membrane with all its sides anchored (A-A-A-A) was built to compare the behaviour of two devices with the same manufacture process but with different boundary conditions.

FEM modelling prediction of the designs with different boundary conditions is used to explain the results of the mechanical (interferometric displacement measurement) and electrical (electric impedance) tests of the probes from the first run. A criticism of the different typologies is given addressing the future transducer designs.

\section{FABRICATION PROCESS}

PolyMUMPs is a standard manufacture process which has the following features: three doped polysilicon layers are used as the structural material, deposited oxide (PSG) is used as the sacrificial layer, and silicon nitride is used as electrical isolation between the polysilicon and the heavily doped silicon substrate. The design rules of the process are described in [5].

Fig. 1 shows the configurations and the dimensions of the layers used to design each typology described in this paper. The first configuration (Fig.1.a) was used in a previous work made in the Carnegie Mellon University of Pittsburgh [6]. The difference with the present designs lies in the shape, the boundary conditions and the size of the membrane. This configuration was chosen to study the differences between the two kinds of boundary conditions: F-A-F-A and A-A-A-A. It uses the polysilicon layer POLY0 as the bottom electrode and the POLY1 as the membrane and top electrode. The rest of the models use the heavily doped silicon substrate as the bottom electrode. These typologies use F-A-F-A boundary conditions. In the case of the si-METAL design two sizes of gold electrode (20 $\mu \mathrm{m}$ and $75 \mu \mathrm{m}$ ) were used to compare the effect of the metallization of the polysilicon layer POLY2. 


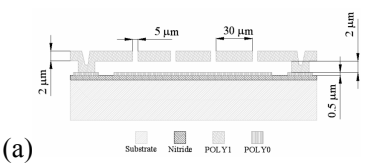

(a)

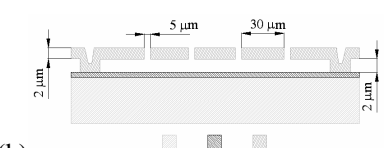

(b)
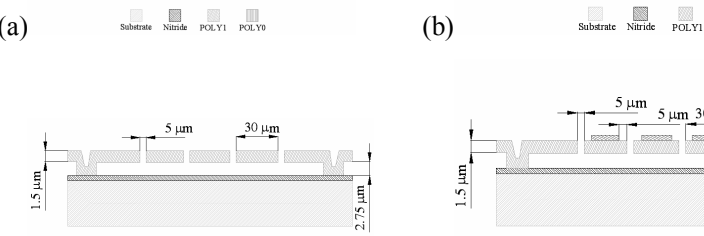

(c)

Substrate Nitide POL.Y.

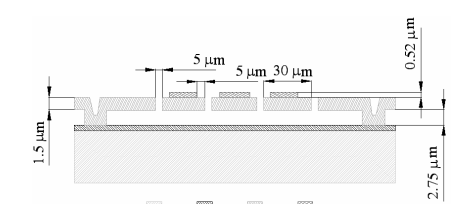

(d)

Figure 1. Cross sections of the cMUT cells. (a) POLY0-POLY1. (b) SiPOLY1. (c) Si-POLY2. (d) Si-METAL

\section{FEM CALCULATIONS}

The finite element models of the designs which use the POLY0-POLY1 configuration was build using the commercial package ANSYS. They were used to identify the mechanical losses of each model. The model was fitted through the simulation of the membrane displacement per frequency. The TRANS126 element was used to model the air gap. It converts energy from an electrostatic domain into a structural domain and vice versa. To model the membrane, the SOLID45 structural element was used. Each model was reduced to the membrane and air gap. As the bottom electrode does not move, it was modeled by the bottom nodes of the TRANS126 elements. The material properties of the polysilicon are listed in Table I.

A pre-stressed harmonic analysis was performed to fit the model. The first step is to obtain the displacement of the membrane through a static analysis in which a polarization voltage of 40 volts is applied. Then, using the PSTRES,ON command, this static solution is used as the starting point for a modal analysis to obtain the resonant frequencies of the membrane. Finally, a voltage of 3 volts amplitude is applied performing a harmonic analysis around the first resonant frequency. Fig. 2 shows how the simulations are fitted to the experimental results.

\section{EXPERIMENTAL SETUP}

The characterization of the devices was performed in the ACULAB - Dip. Ing. Elettronica of the Università Roma Tre in Italy. An HP 4194A impedance/gain-phase analyzer was used to measure the real part of the electrical impedance per frequency. The measurements were done using a polarization voltage of 40 volts DC and a signal of 1 volt AC peak to peak.

TABLE I. MATERIAL Properties

\begin{tabular}{ccc}
\hline & Polysilicon & Air \\
\hline Young's Modulus (GPa) & 165 & \\
Poisson's Ratio & 0,22 & \\
Density (Kg/m ' $\left.^{3}\right)$ & 2330 & \\
Relative Permittivity & & 1,04 \\
\hline
\end{tabular}
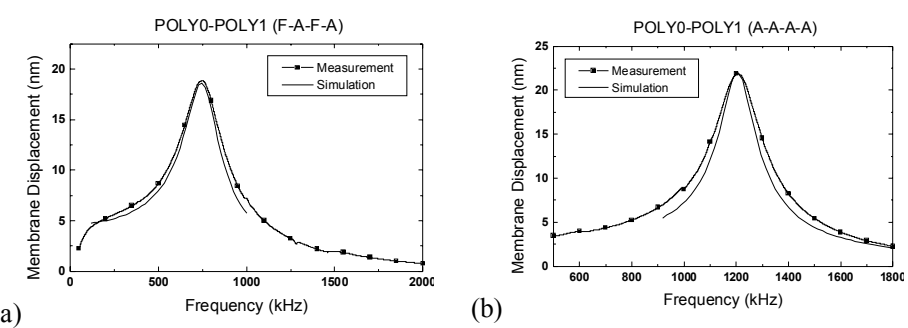

Figure 2. Experimental result and simulation of the displacement of a membrane with F-A-F-A (a) and A-A-A-A (b) boundary conditions.

Once the resonant frequency of each membrane was known, a Laser vibrometer Polytec MSV 300 was used to measure the displacement of each membrane. The Polytec MSV 300 consists of a scanner controller MSV-Z-040, microscope adapter 0FV 074 and video camera, microscope scanner unit OFV 073, interferometric unit OFV 511 and a vibrometer controller OFV 2700-2 with a displacement decoder. The DMLMP/ Leica microscope was used to monitor the position of the measure and as a part of the interferometric system. Array elements of $29 \times 2$ cMUT cells of each different design were the devices to test. They were excited with an AC voltage of 3 volts amplitude supplied by a HP8116A function generator on top of 40 volts DC polarization voltage. A TDS5034B Tektronix oscilloscope was the device used to capture the signal and finally a personal computer was used to process all the data from the TDS5034B. All this system was required to see the displacement at the centre of the membrane per frequency and the deformed shape of each device at the resonant frequency.

\section{EXPERIMENTAL RESULTS}

To study which cMUT cell is the best option to develop the future air-coupled transducer, an electrical and a mechanical characterization of each kind of array element was performed.

\section{A. Electrical Characterization}

The real part of the impedance of the transducer was measured to view the resonant frequency and to study the losses of each design. The equivalent circuit model in Fig. 3 is used to understand the measures of this section. The equivalent impedance of the circuit can be written as follows:
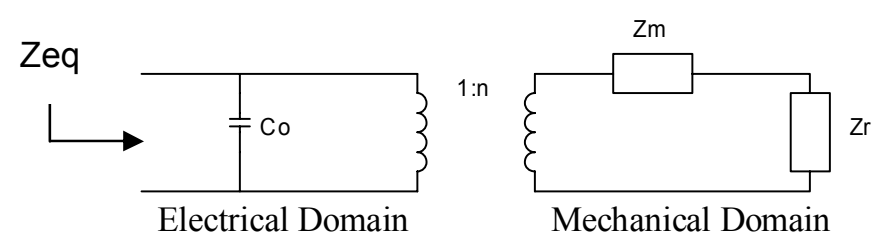

Figure 3. Equivalent circuit model for a transmiting cMUT 


$$
Z e q=\frac{1}{Y e q}
$$

Where,

$$
Y e q=G e q+j \cdot B e q
$$

From the circuit the following equation is obtained:

$$
\begin{aligned}
& Y e q=j \cdot \omega \cdot C_{0}+\frac{1}{\left(\frac{Z m+Z r}{n^{2}}\right)}=\frac{1}{R m^{\prime}+R r^{\prime}+j \cdot\left(X m^{\prime}+X r^{\prime}\right)} \\
& +j \cdot\left(\omega \cdot C_{0}-\frac{X m^{\prime}+X r^{\prime}}{\left(R m^{\prime}+R r^{\prime}\right)^{2}+\left(X m^{\prime}+X r^{\prime}\right)^{2}}\right)
\end{aligned}
$$

Where $R m^{\prime}, R r^{\prime}, X m^{\prime}$ and $X r^{\prime}$ are the real and imaginary parts of $Z m$ and $Z r$ divided by $n^{2}$.

From the following expression:

$$
\operatorname{Re}\{Z e q\}=\frac{G e q}{G e q^{2}+B e q^{2}}
$$

And taking advantage of the fact that at the resonant frequency $\left(X m^{\prime}+X r^{\prime}\right)$ is approximately zero, (4) can be rewritten using (3) yielding:

$$
\left.\operatorname{Re}\{Z e q\}\right|_{\omega=\omega_{0}} \cong \frac{R m^{\prime}+R r^{\prime}}{1+\left(\omega_{0} \cdot C_{0}\right)^{2} \cdot\left(R m^{\prime}+R r^{\prime}\right)^{2}}
$$

Analyzing this equation, it is shown that the real part of the impedance at its resonant frequency does not depend on the variations of the fixed capacitance $\left(C_{0}\right)$ and the frequency. It varies linearly with the mechanical losses $\left(R m^{\prime}+R r\right)$ at the range of the measured values of $\operatorname{Re}\{Z e q\}$ with the impedance analyzer. At this range, $\left(\omega_{0} \cdot C_{0}\right)^{2} \cdot\left(R m^{\prime}+R r^{\prime}\right)^{2}$ is much lower than 1 . This result will be used to delimit the losses of the next measures.

Fig. 4 shows the resistance per frequency of each design. In all cases it is shown that the resistance is on top of a negative exponential due to electrical losses. A study of the effects of this fact will be needed in a future work. Fig. 4.a shows a comparison between the designs which use F-A-F-A boundary conditions and the POLY1 layer as the membrane and top electrode. The resonances have almost the same values of amplitude and width in both cases. This means that the mechanical losses are approximately the same. The displacement results of the next section show what design has more radiation losses.

The comparison between the designs with the same combination of layers (POLY0-POLY1) but with different boundary conditions (F-A-F-A and A-A-A-A) is shown in Fig. 4.b. The A-A-A-A has its resonant frequency at approximately 1.2 MHz. The amplitude of the resonance in this case is lower than the corresponding value of the F-A-F-A. This is due to the different mechanical losses of these designs. This fact can be explained using the FEM model and the equivalent circuit model. To fit the FEM simulation with the experimental displacement in the A-A-A-A case, a lower damping factor was introduced. On the other hand, for this frequency range and for the values of the fixed capacitances of each design, the peak of the resonance depends on the value of $\left(R m^{\prime}+R r^{\prime}\right)$. This means that the F-A-F-A design has higher mechanical losses. However, it must be taken into account the deformed shape and the displacement of the membrane to study the most efficient option.

Fig. 4.c shows the frequency response of the resistance of the designs which use the silicon substrate as the bottom electrode but do not use metallization (Si-POLY1 and SiPOLY2). The Si-POLY2 has the smallest resonance and it is situated at approximately $470 \mathrm{kHz}$. This is due to its gap which consist of $2.75 \mu \mathrm{m}$ of the removed sacrificial oxide layers and $0.6 \mu \mathrm{m}$ of the silicon nitride isolation layer. However, the POLY2 membrane has the smallest thickness $(0.52 \mu \mathrm{m})$ and it is the one which can be covered by the gold metallization. The effect of the POLY2 metallization is shown in Fig. 4.d. As the size of the metal electrode increases, the amplitude of the resonance increases proportionally and the resonant frequency shifts to a lower values. In the next section it can be seen additional effects of the metallization and the displacement of the membrane in each case.

\section{B. Mechanical Characterization}

To study the behavior of the membranes of each array element, an interferometric displacement measure was performed. Fig. 5.a shows the displacement per frequency of the designs which use the POLY1 as the membrane and top electrode. As it is said in the previous section, the resonances of these F-A-F-A designs are almost the same. The main difference is due to the gap dimensions. In the Si-POLY1 design, the thickness of the silicon nitride layer is added to the total gap size. In the case of the A-A-A-A design, the displacement at the centre of the membrane is higher than that of the other designs with the POLY1 as the membrane. In the previous section was demonstrated that the F-A-F-A design has more losses. This displacement curve shows that the $\mathrm{Rm}$ part of the mechanical losses might be higher in the F-A-F-A. (a)

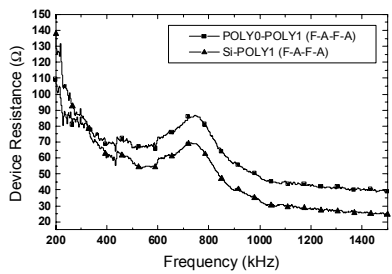

(c)

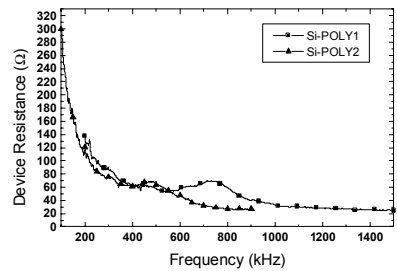

(b)

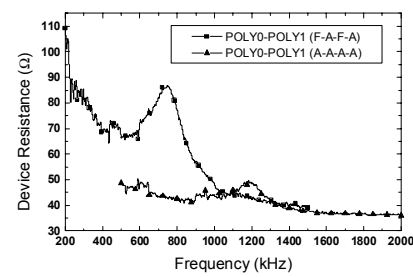

(d)

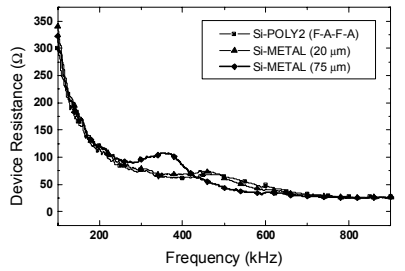

Figure 4. Real part of the impedance $(\Omega)$ of each design: (a) POLY0POLY1(F-A-F-A) \& Si-POLY1; (b) POLY0-POLY1 F-A-F-A \& A-A-A-A; (c) Si-POLY1 \& Si-POLY2 (F-A-F-A); (d) Si-POLY2 \& Si-METAL $(20 \mu \mathrm{m}$ and $75 \mu \mathrm{m})$ 
(a)

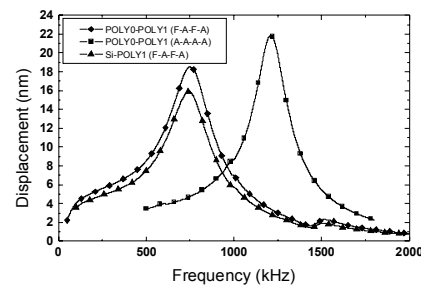

(b)

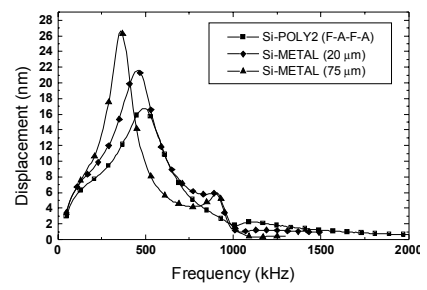

Figure 5. Frequency response of the displacement of each membrane: (a) POLY0-POLY1 (F-A-F-A \& A-A-A-A) and Si-POLY1; (b) Si-POLY2 and Si-METAL $(20 \mu \mathrm{m} \& 75 \mu \mathrm{m})$

However, as it is shown in Fig. 6 the integration surface in the F-A-F-A designs is bigger than the A-A-A-A design which has its maximum displacement only at the centre of the membrane. Then, using the F-A-F-A boundary conditions more ultrasonic energy will be emitted through the air for the same displacement. This explains why the peak of the resonance of the F-A-F-A design in Fig. 4.b is higher than that of the A-A-A-A because of the radiation losses $\left(R r^{\prime}\right)$ in spite of the value of $R m^{\prime}$. However, it is necessary an analytical study of the mechanical losses such as the effects of the spring constant.

Fig. 5.b shows the displacement per frequency of the designs which use the POLY2 as the membrane. As it was (a)

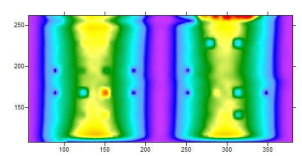

(c)

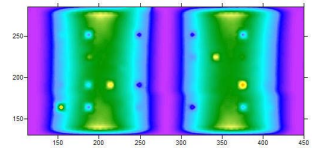

(e)

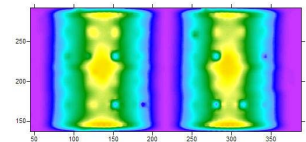

(b)

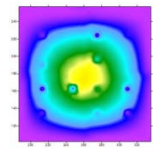

(d)

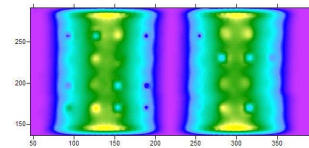

(f)

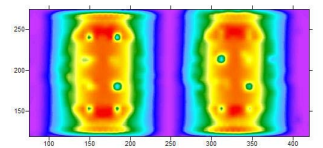

Figure 6. Displacement map of each membrane at their resonant frequency (scale nm): (a) POLY0-POLY1 (F-A-F-A); (b) POLY0-POLY1 (A-A-A-A); (c) Si-POLY1 (F-A-F-A); Si-POLY2 (F-A-F-A); Si-METAL $(20 \mu \mathrm{m})(\mathrm{F}-\mathrm{A}-\mathrm{F}-$ A); ); Si-METAL $(75 \mu \mathrm{m})(\mathrm{F}-\mathrm{A}-\mathrm{F}-\mathrm{A})$ said in the previous section, the addition of the metallization causes a shift in the resonant frequency. However, it is shown how the displacement becomes higher as the electrode size increases. This explains that the real part of the impedance is higher in the design with a bigger electrode. The radiation losses $\left(R r^{\prime}\right)$ are higher in this case. In spite of these affirmations, there is a fact which needs a further study. In the designs with metallization, a second resonance appears approximately at $910 \mathrm{kHz}$. This resonance is near from the first resonant frequency and it might affect the behavior of the membrane. In Fig. 6.d, Fig. 6.e and Fig. 6.f a displacement map of these membranes are shown. Here the Si-METAL designs do not seem to be affected by this effect. An accurate FEM model will be needed in a future work to study carefully the effects of this metallization.

\section{CONCLUSIONS}

A presentation of several designs of cMUT cells manufactured using the MUMPs standard process is described in this paper. The results of the electrical and mechanical characterization of several cMUT array elements of $29 \times 2$ cells and the FEM simulation of two single cMUTs show that the designs which use the special boundary conditions F-A-F-A will work better in spite of their mechanical losses. A further work is needed to identify these losses and to understand the effects of the metallization of the POLY2 layer.

\section{ACKNOWLEDGMENT}

The authors are grateful to the staff at the ACULAB - Dip. Ing. Elettronica of the Università Roma Tre in Italy for their help during the measurements.

\section{REFERENCES}

[1] D.A. Hutchins and D.W. Schindel, "Advances in Non-contact and Aircoupled Transducers”, Proc. 1994 IEEE Ultrasonics Symposium, 1994, pp 1245-1254

[2] A. J. Rogovsky. "Development and application of ultrasonic dry-contact and air-contact c-scan systems for nondustructive evaluation". Materials Evaluation pages, pp 1491-1497, December 1991.

[3] M. I. Haller, B. T. Khuri-Yakub " A Surface Micromachined Electrostatic Ultrasonic Air Transducer", IEEE Trans. on UFFC, vol. 43, pp. 1 - 6, Jan. 1996.

[4] X.C. Jin, I. Ladabaum, F.L. Degertekin, S. Calmes and B.T. KhuriYakub, "Fabrication and Characterization of Surface Micromachined Capacitive Ultrasonic Immersion Transducers," IEEE/ASME Journal of Microelectromechanical Systems (JMEMS), 8, pp. 100-114, 1999.

[5] Jim Carter, Allen Cowen, Busbee Hardy, Ramaswamy Mahadevan, Mark Stonefield and Steve Wilcenski, "Poly MUMPs Design Handbook" MEMSCAP Inc. Revision 11.0.

[6] Irving J. Oppenheim, Akash Jain and David W. Greve, "Electrical Characterization of Coupled and Uncoupled MEMS Ultrasonic Transducers", IEEE Transactions on Ultrasonics, Ferroelectrics, and Frequency Control, Vol. 50, N 3, March 2003. 